\title{
Glioblastoma Drug Treatments and Animal Models
}

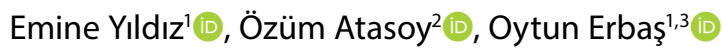

Glioblastoma multiforme (GBM) is the most common and deadliest brain tumor among the primary neoplasms of the brain. ${ }^{[1]}$ Glioblastoma has a primary or secondary origin and the tumor can spread and reach advanced stages. In addition to cytogenetic differences in the primary and secondary stages of GBM, characteristic structures in genetic pathways have also been identified in GBM. It has been shown that GBM is mostly seen in adults and extremely rare in children, accounting for no more than $3-15 \%$ of primary central nervous system (CNS) tumors. Likewise, it is a highly mortal disease in childhood. ${ }^{[2]}$

Although imaging techniques are important for the diagnosis of the tumor seen in both adults and children, the appearance of symptoms is the first step for the patient. Most patients with glioblastoma have a history of symptoms ranging from 3 to 6 months, but if the tumor arises from a low-grade astrocytoma, the patient's history and symptoms can spread up to several years. ${ }^{[3]}$ The source of these symptoms is brain tissue damage as a result of necrosis caused by cognitive impairments. The area of the patient's complains can actually be related to

'ERBAS Institute of Experimental Medicine, Illinois, USA \& Gebze, Turkey ${ }^{2}$ Department of Radiation Oncology, Kartal City Hospital, Istanbul, Turkey ${ }^{3}$ Department of Physiology, Medical Faculty of Demiroğlu Bilim University, Istanbul, Turkey

Correspondence: Emine Yıldız. Deneysel Tıp Enstitüsü, 41470 Gebze-Kocaeli, Türkiye.

E-mail: emmoyildiz@gmail.com

Cite this article as: Yıldız E, Atasoy Ö, Erbaş O. Glioblastoma Drug

Treatments and Animal Models. JEB Med Sci 2020;1(3):140-146.

doi: $10.5606 /$ jebms. 2020.75630

Received : October 04, 2020

Accepted : October 26, 2020

Published online : December 29, 2020

@2020 Journal of Experimental and Basic Medical Sciences. All rights reserved.

\begin{abstract}
Glioblastoma is a malignant tumor that is seen in the cerebral hemispheres, cerebellum, spinal cord and brain stem. This tumor, which follows an invasive approach, consists of the cells called astrocytes that form the most important part of the glia cells. When the disease arises, the neurons are directly affected and neurotransmitter substances cannot fulfill their functions, as well as a memory damage may occur. Studies have been conducted on experimental animals in order to perform better analysis in the treatment for years. As a result of studies on rat and mouse models, it has been proven that drugs such as irinotecan, bevacizumab, temozolomide and their active ingredients are promising for patients. In this review, it is aimed to reveal how glioblastoma emerged, its diseases and symptoms by working step by step on animal models of rats and mice, and to expand the field of drug treatment. Keywords: Glia, glioblastoma, neuron.
\end{abstract}

the lobes where the tumor is formed. For example, tumor formation is observed in the temporal lobe of patients with visual and hearing difficulties while a tumor formation is observed in the frontal lobe as a result of personality disorder or change. ${ }^{[3,4]}$ If the mass area covered by the tumor is large, motor deficits may also occur in the patient. ${ }^{[5]}$ The internal pressure in the head increases with the increase in the size of the tumor and the increase in tissue edema. This would be resulted with headache, which is the biggest complaint of glioblastoma patients. ${ }^{[3,4]}$ While this pain usually occurs in one part of the head, vomiting is also observed due to an increased pressure ${ }^{[5]}$ Epileptic seizures are also quite common in patients, depending on the location of the tumor. ${ }^{[3,5]}$

Imaging techniques are used to diagnose the patient with a complete brain tumor. These techniques mainly are computed tomography (CT) and magnetic resonance imaging (MRI) scans. With these imaging techniques, information has been 
acquired from the tissues, cell structures, metabolism and hemodynamics of gliomas in recent years and these have been evaluated with the patient's history. Apart from these, positron emission tomography (PET) and single photon emission computed tomography (SPECT), which are nuclear medicine techniques, are the techniques used to analyze and separate the changes related to the treatment with active tumor. ${ }^{[6]}$

Along with the patient's complaints and the imaging technique performed on it, a brain tumor is diagnosed in the foreground and a definitive diagnosis is made according to which cell the tumor originates from after the pathological evaluation. Concomitant and adjuvant chemoradiotherapy with oral temozolamide (TMZ) after maximal safe surgical resection is a standard in the treatment. In addition, having an idea in the genetic structure and characteristics of the tumor with the developing technology and applying treatment accordingly is a promising approach in terms of the development of new treatment modalities.

\section{GENETIC PROPERTIES OF GLIOBLASTOMA MULTIFORM}

When looking at the molecular structure of glioblastoma, it is possible to collect it under two headings. These; primary glioblastoma and secondary glioblastoma. Most cases (> 90\%) form primary glioblastomas that develop rapidly de-novo. It is often seen in older people. ${ }^{[7]}$ Epidermal growth factor receptor (EGFR) mutation is quite common in the primary glioblastoma process. ${ }^{[7]}$ Members of this family and the key-key ligands they connect with have played an important role in central nervous system cell differentiation and migration. ${ }^{[8]}$
EGFR gene amplification (40-80\%) and overexpression (10-60\%) are seen in GBM. ${ }^{[9]}$ Seven mutant variants of the EGFR gene have been identified, the most observed variant III (EGFRvIII), also called de2-7EGFR or $\rho$ EGFR. EGFRvill occurs as a result of deletion in exons 2-7. As a result of this genomic deletion, the key lock match is not achieved, resulting in the lack of the extracellular ligand binding site, the receptor fails to function and tumor growth, proliferation, and tumor invasion are also increased. In addition, the characteristic structure of the tumor is formed with the P16INK4A deletion (31\%). ${ }^{[10]}$ Other distinctive changes include phosphatase, which keeps chromosome 10q, and a loss of heterozygosity.

(LOH) of the tensin homolog (PTEN), and TERT promoter mutation is also observed. ${ }^{[2]}$

Secondary GBM develops from low grade diffuse astrocytoma or stage III anaplastic astrocytoma. It takes about 5 years for astrocytomas with a low grade to turn into a true GBM. ${ }^{[1]}$ Secondary GBM, unlike primary GBM, is seen in younger ages, on average in the $40 \mathrm{~s}$.

For the diagnosis of secondary glioblastoma, unlike primary glioblastoma, a histological finding is required beforehand, and a TP53 mutation is seen on the genetic basis, which can be detected in the first course of the disease. ${ }^{[7,12]}$ Another important finding is the overexpression of thrombocyte-derived growth factor $A$.

In addition, mutations in cyclin-dependent kinase inhibitor 2 (CDKN2A) genes and overexpression of MDM2 protein are common genetic changes. ${ }^{[11]}$ TP53 pathway plays a critical
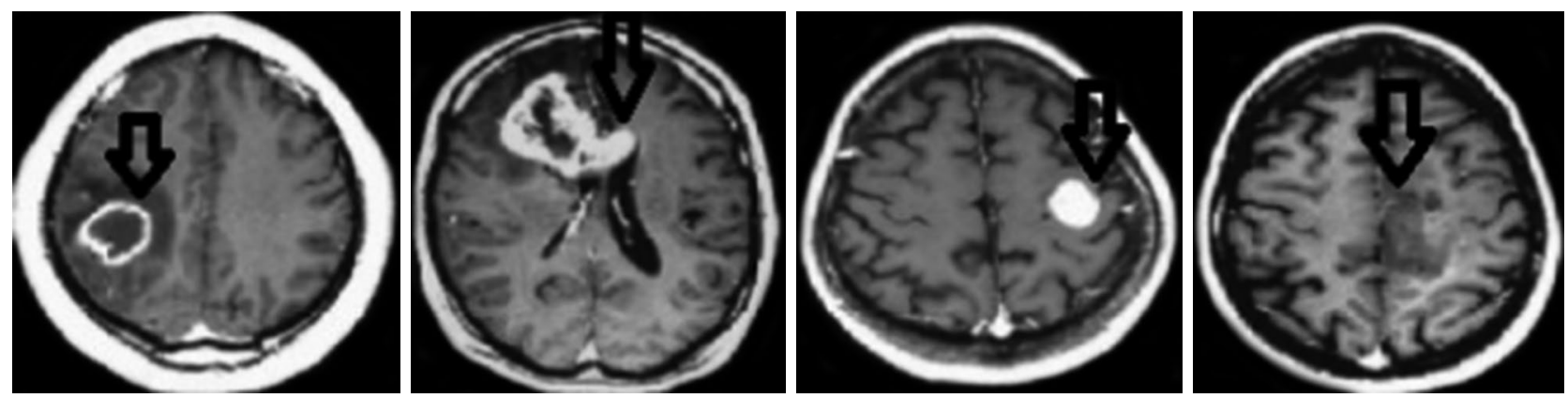

Figure 1. Four different patients with glioblastoma multiforme showing heterogeneity in an anatomical lesion. In the first patient, central necrosis was observed in the right parietal lobe with edema. In the second patient, an irregularly growing mass passing through the corpus callosum was detected. The third patient also had a well-circumscribed, homogeneously enhancing mass in the left frontal lobe without associated edema. Patient 4 showed a poorly defined infiltrative mass in the left medial frontal lobe without significant necrosis. Although 4 patients had different levels of edema or necrosis in the brain, it was found that the common denominator was glioblastoma. 
role in the development of secondary glioblastoma. TP53 gene encodes a protein that has a prominant role in many cellular processes including cellular response to DNA damage, cell death and cell differentiation. ${ }^{[7,12]}$ When the mechanism of this protein is disrupted, tumor formation will be inevitable because DNA damage cannot be repaired. In addition, p53 mutations in secondary GBMs are seen at higher rates than $65 \%$.

In addition to all these molecular and genetic events, chromosome 10 loss $^{[7]}$ and tumor suppressor PTEN gene deletion are the common genetic changes in primary and secondary GBM. Genetic changes such as TP53 mutation and CDK4 amplification are encountered in both types of GBM. ${ }^{[13,14]}$

In tumor cells seen in the tissue of glioblastoma, cystic degeneration, increase in signals, angiogenesis, uncontrolled and rapid division and proliferation, an invasive as a result, the cell becomes resistant to apoptosis. ${ }^{[15,16]}$ In a healthy brain, while all these stages are controlled by signaling pathways and the system is maintained, the functioning of some genes and receptors is disrupted which will result with edema in the tumor and its surroundings, and signal pathways become inoperable.

Another important pathway detected in GBM cells and triggering viability is the phosphatidylinositol 3 kinase (PI3K) pathway. As a result of the activation of various tyrosine kinase receptors (such as EGFR, PDGFR) with growth factors, the PI3K / Protein Kinase $B(P K B, A K T)$ pathway is activated and it leads to the genes that regulate the proliferation of cells are activated. PTEN can block this pathway, however, it has been stated that the PTEN mutation detected in GBM does not inactivate this pathway. Another pathway triggered by the activation of tyrosine kinases is the mitogen activating protein kinase (MAPK) pathway via the RAS oncogene. Activation of this pathway is associated with the activation of transcription factors that enable the expression of the genes that regulate glial cells growth. Mutation in tyrosine kinase receptors that cause the activation of these signals in GBM causes uncontrolled activation of signal pathways. ${ }^{[10]}$ As a result, cells divide and differentiate in an uncontrolled manner, ultimately, it will lead to the formation of a tumor.

Another important molecular finding is that DNA methylation directly affects GBM, because DNA methylation consists of an epigenetic mechanism. Aging is of great importance in cancer and genomic suppression factors. ${ }^{[17]}$ Hypomethylation in tumor cells has led to the loss of methylation in the repeating regions of the genome and the reactivation of genes. Here, the loss of methylation affected the course of the disease in relation to the type of tumor. ${ }^{[18,19]}$ As a result of hypermethylation of faulty cytosine units in the promoter regions, tumor suppressor genes became silenced and DNA repair genes became dysfunctional. A series of problems are encountered when DNA repair genes cannot repair the damaged area. $^{[17,10]}$

Therefore, it is understood that chromosomal losses are generally more than chromosomal gains in GBM. The GWAS studies carried out in gliomas since 2009, it has been shown that polymorphisms in 8 different genomic regions are associated with glioma susceptibility. ${ }^{[2]}$ These genomic regions are: TERC (rs1920116), TERT (rs2736100), EGFR (rs2252586, rs11979158), CCDC26 (rs55705857), CDKN2B (rs1412829), PHLDB1 (rs498872), TP53 (rs78378222, rs), TP53 (rs78378222), rs60 23).

For example, while CCDC26 polymorphisms do not show a significant effect on glioblastoma risk, it has been proven that the presence of the SNP rs55705857-G allele located in the CCDC26 gene increases the risk of isocitrate dehydrogenase (IDH)mutant glioma by 16 -fold, and this is achieved through the interaction of the MYC oncogene with remote region DNA, which is approximately 2 million base pairs away. Likewise, it has been proven that PHLDB1 polymorphisms increase the risk of developing IDH mutant glioma, whereas CDKN2B polymorphisms, TERC polymorphisms, astrocytoma and glioblastoma. ${ }^{[2]]}$ The IDH mutation aforementioned is one of the biggest mutations that cause Glioblastoma disease.

\section{ISOCITRATE DEHYDROGENASE MUTATION}

Isocitrate dehydrogenase (IDH) is a gene that codes for the enzyme that catalyzes the conversion of isocitrate to alpha-ketogluterate. The mutations in this gene results with the function of the enzyme deteriorates and causes 2-hydroxygluterate (2-HG), which is an oncometabolite, to be produced excessively in the cell. ${ }^{[22]}$ Subsequently, 2-HG increases the expression of protooncogenes by stimulating the epithelium-mesenchymal transition (EMT), thus inhibiting dioxygenases, causing DNA and histone modifications and alteration of the cell microenvironment, causing damage to the cell and its environment. ${ }^{[23]}$ Studies have found an evidence that IDH mutations contribute to the formation of glioblastoma. The main task of IDH is to protect the 
cell against oxidative stress that may occur. And, the mutation that occurs in renders cells vulnerable to oxidative stress. ${ }^{[24]}$

When looking at the human genome, it is known that it has 3 different IDH enzymes (IDH1, IDH2, IDH3) and $5 \mathrm{IDH}$ genes encoding them; IDH1, IDH2, IDH3A, IDH3B and IDH3G genes. IDH1 is located in the cytoplasm and peroxisome whereas IDH2 and IDH3 are located in the mitochondria. ${ }^{[25,26]}$ It is inevitable that other cells will be affected by this situation when they mutate within the cell due to their location. We can say that IDHs have an important role in cellular metabolism. IDH1 is involved in the metabolism of fats and glucose tolerance, regulation of $\mathrm{IDH}_{2}$ oxidative respiration, and aerobic energy production in the IDH3 tricarboxylic acid cycle. ${ }^{[26]}$

As a result of mutations caused by IDH1 in gliomablastoma, it has been determined that the $\mathrm{R} 132 \mathrm{H}$ mutation causes arginine amino acid to histidine with (G395A), and IDH2 causes the conversion of arginine to lysine with the R172K mutation (G515A). ${ }^{[27]}$ The IDH1 mutation is specific for each tumor, and IDH1 mutation has been observed in various types of glioma, especially in histologically classified low-grade gliomas and secondary glioblastomas. ${ }^{[26]}$

As a result of the overexpression of IDH1, the protection against to the reactive oxygen species upregulated, radiation increases and supports oxidative stress, while IDH1 inhibition causes a decrease in cell protection mechansim and leads to an increase in tumor growth and angiogenesis. ${ }^{[28]}$

\section{GLIOBLASTOMA MODELS}

\section{Mouse models}

A glioma model induced by injections of N-Ethyl$\mathrm{N}$-Nitroside (ENU) was created. This model was formed in the late 1970s and created by intravenous injection of ENU. ENU is given to pregnant rats on day 18. In the model, it is tried to mimic the tumors seen in humans as a result of genetic instability, p53 or mutations in the genes that enable the progression of the cell cycle. In this way, the ENUinduced model exhibits the properties similar to the human glioma in several ways, including, tumor formation and environment, active immune system, and intact blood-brain barrier. However, it is a costly modeling, but, still results have been obtained. ${ }^{[29]}$ In another experiment, its ENU was dissolved in phosphate buffer adjusted to $\mathrm{pH} 6$. It was injected intraperitoneally into the mouse in $1 \mathrm{ml}$.
Wild-type male 9-19 week old mice were injected with a single dose. $(250 \mathrm{mg} / \mathrm{kg})$ For this given dose rate, all animals survived while the dose given to a $300 \mathrm{mg} / \mathrm{kg}$ mouse killed about $40 \%$. At the end of the experiment, mice also showed dominant skeletal mutations, and it was also found that the males were fertile in mating for about 3 weeks after injection, then entered a transient sterile period.

Genetically modified mice have been used as animal models for human neoplasms to investigate tumor formation and the impression of the tumor in an intact organism. Weissenberger constructed a transgenic mouse model showing overexpression of the v-src oncogene under the control of the GFAP regulatory promoter. Transgenic mice developed low-grade astrocytomas and glioma in the early phase, while the advanced phase produced highgrade astrocytomas. The GBM model was created with high mitotic activity as a result of necrosis. ${ }^{[30]}$

One of the other transgenic models created in mice is the Cre-Lox model that is known as the most classic example. Lox $\mathrm{P}$ transgenic mice overexpressing the oncogene, Ras, and conditionally not containing the tumor suppressor gene, p53, are used. Cre recombinase is usually placed under the glial cell-specific promoter as GFAP and delivered to the mouse. The driver mutations information is required to design the Cre-Lox system. The purpose of this model is to investigate the efficacy of drug compounds with predefined genetic structure. ${ }^{[31]}$ The advantage of this model is to be able to analyze the microenvironment, which will define the molecular events responsible for tumor initiation and progression. The disadvantage, however, is that it does not fully reflect intratumoral genomic and phenotypic heterogeneity, i.e. the onset of the tumor cannot be fully controlled.

Another model is the murine allograft model. It is generated by intracranial injection of glioma cell lines of murine origin (as C6) in Wistar rats or C57BL/6 mice. The advantage of the model is that it allows cell lines to be orthotopically implanted in animals with active immune systems. Allogeneic immune response is observed in wistar rats with low tumor growth. ${ }^{[31]}$ Tumors that develop after implantation are often found invasive. In this model, the effects of targeted treatments are investigated.

Syngeneic mouse models of glioblastoma have long been indispensable models for glioblastoma research. These models (GL261, GL26, CT-2A and P560) were created from spontaneously or chemically induced murine glioma. GL261, GL26 
and CT-2A are derived from chemically induced glioblastoma mouse models while P560 is derived from spontaneous mouse models of glioblastoma. GL261 models are the most commonly used model to create glioblastoma. Because these models are known to have the most suitable structure for the histological and biological features of glioblastoma. In addition, these models also utilize immunocompetent mice and have therefore been chosen as the most suitable for glioblastoma tumor immunology and immunotherapeutic studies. ${ }^{[32]}$

However, PDX, GEM, and syngeneic models include the best clinical and biological mouse model studies for glioblastoma. The disadvantages of genegenerated mouse models for glioblastoma may include few as they may not completely recapitulate the pathology of human-origin tumors. Also, the course of targeted monoclonal antibodies may not mimic due to the difference in the complete set of immunological reactions between human and mouse/rat.

\section{Rat model}

Changes in glial cells were observed in transgenic models created by infusing human tumor cells (U87) into the frontal lobe of Sprague-Dawley rats. When the histopotological structure of the brain was examined, there have been higly excessive pleomorphism, mitotic activity, and necrosis structure observed in the cells. In addition, it was observed that the immunity decreased in rats given U87. ${ }^{[33]}$

\section{GLIOBLASTOMA TREATMENT}

\section{İrinotecan}

The active metabolite of irinotecan is 7-ethyl-10hydroxycamptothecin ( $\mathrm{SN}-38$ ). Irinotecan is a specific inhibitor of DNA topoisomerase I. The glioma cell can convert irinotecan directly to SN-38. Increasing $\mathrm{SN}-38$ concentrations induce morphologically cytotoxic changes, reduce proliferation and increase cytotoxicity. The mechanism of cytotoxicity is apoptosis. In studies, SN-38 caused a decrease in antiapoptotic protein $\mathrm{Bcl}-2$ concentration and an increase in proapoptotic protein Bax expression.

It is a camptothecin derivative that inhibits topoisomerase $\mathrm{l}$, an essential nuclear enzyme required for the relaxation of supercoiled DNA, providing topological changes that facilitate RNA transcription and DNA replication. ${ }^{[34]}$

Topoisomerase I and II activities are significantly increased in malignant gliomas following DNA damage. Chromatin-bound topoisomerase I and II levels are associated with induction of apoptosis by DNA-damaging agents, and induction of apoptosis is associated with a decrease in $\mathrm{BCl}-2$. showed counter activity. ${ }^{[35]}$

Although its usage varies depending on the type of glioblastoma, the recommended dosage for a single substance is $125 \mathrm{mg} / \mathrm{m}^{2}$. According to the studies, when Irinotecan $\left(125 \mathrm{mg} / \mathrm{m}^{2}\right.$ in relapsed or malignant progressive adult patients) was used in 6 cycles once a week for 4 weeks, more than 50\% reduction in tumor size was detected.

\section{Bevacizumab}

Vascular Endothelial Growth Factor (VEGF) is a signal protein produced by cells that stimulate angiogenesis. Bevacizumab, a humanized monoclonal antibody that targets VEGF, has been used in patients with brain tumor due to intracranial hemorrhage. A series of 29 patients with malignant glioma treated with bevacizumab and irinotecan were studied in a combination of bevacizumab and irinotecan due to a significant bleeding, and single arm phase 2 trials for GBM, with a rate of $57 \%$ and progression-free survival rates of $46 \%$ at 6 months. ${ }^{[36,37]}$

Bevacizumab is generally well tolerated, with the most common side effects being mild bleeding such as hypertension and epistaxis. It has also been found that irinotecan does not increase the efficacy of single agent bevacizumab in recurrent malignant gliomas and has significant toxicity effects such as diarrhea and myelosuppression. The result of this treatment-responsive study of bevacizumab as a single agent in recurrent GBM has increased interest in evaluating the use of a drug as an initial therapy for newly diagnosed GBM patients.

\section{Temozolomide}

Temozolomide is used in the treatment of glioblastomas. Temozolomide is a prodrug; it is spontaneously hydrolyzed at physiological $\mathrm{pH}$ to 3-methyl-(triazen-1-yl)imidazole-4-carboxamide (MTIC). MTIC splits into monomethylhydrazine, probably the active methylating agent, and 5-aminoimidazole-4-carboxamide (AIC). Therefore, temozolomide does not require hepatic metabolism for activation.

The interest with temozolomide as an antitumor agent is due to its broad-spectrum antitumor activity in tumor models in mice. Temozolomide has demonstrated cell-dependent antitumor activity against a variety of malignancies, including glioma, 
and other difficult-to-treat cancers in vitro. The tumor has been found to shrink and its effects are diminished. It has generally shown an antitumor activity against a wide range of tumors, including glioma, melanoma, mesothelioma, sarcoma, lymphoma, leukemia, and colon and ovarian carcinoma. Its ability to cross the blood-brain barrier has played an important role due to its activity on central nervous system tumors.

The use of temozolomide in the treatment of patients with newly diagnosed malignant glioma has also been evaluated. Thirty-three patients with newly diagnosed glioblastoma multiforme and five patients with anaplastic astrocytoma were treated with an initial dosage of $200 \mathrm{mg} / \mathrm{m}$ given daily temozolomide for 5 days in a 28-day cycle. Responses in $47 \%$ of patients showed reduced tumor reduction and reduced symptoms. ${ }^{[38-40]}$

Based on the results of studies on temozolomide and GBM, temozolomide was approved for the treatment of patients with glioblastoma multiforme that progressed or relapsed after standard therapy and was added to the standard therapy. In the mechanism of action, methylation of DNA appears to be the main mechanism responsible for the cytotoxicity of temozolomide to malignant cells.

\section{Concluding remarks}

Glioblastoma, or known as brain tumor, develops depending on several environmental factor including, radiation, stress, air pollution, living conditions and many other reasons that become a disease that people have to face. Genes are predominantly critical in the formation of brain tumor, which is associated with an inflammation as well as environmental factors. Genetically inherited or subsequent mutations in these genes, such as the IDH mutation, were the triggers of this disease. Although the molecular infrastructure of primary $\mathrm{gbm}$ and secondary gbm diseases is different, their symptoms are the same. Today, transgenic models created on mice are the simplest and easiest models. However, although the rat model has a structure that is more suitable for human tumor cells and may give more precise results, it is noteworthy that fewer studies have been done. Next, different rat models created for glioblastoma will be more useful in developing drugs for the treatment of brain tumor, which has become one of the greatest problems of the world. When the most commonly used drugs such as irinotecan, bevacizumab and temozolomide and radiotherapy cannot be obtained in the combination with each other, drug doses can be increased or a support can be provided with other chemotherapy It is expected that it will become a part of routine treatment with the increase of studies and results on personalized treatment options.

\section{Declaration of conflicting interests}

The authors declared no conflicts of interest with respect to the authorship and/or publication of this article.

\section{Funding}

The authors received no financial support for the research and/or authorship of this article.

\section{REFERENCES}

1. Hanif F, Muzaffar K, Perveen K, Malhi SM, Simjee ShU. Glioblastoma Multiforme: A Review of its Epidemiology and Pathogenesis through Clinical Presentation and Treatment. Asian Pac J Cancer Prev 2017;18:3-9.

2. Aldape K, Zadeh G, Mansouri S, Reifenberger G, von Deimling A. Glioblastoma: pathology, molecular mechanisms and markers. Acta Neuropathol 2015;129:829-48.

3. Clarke CRA. Neurological diseases. In: Kumar P, Clark M, editors. Kumar \& Clark's Clinical Medicine. 6th ed. Edinburgh: Elsevier Saunders; 2005. p. 1244-45.

4. Salah Uddin ABM, Jarmi T. Neurologic manifestations of glioblastoma multiforme clinical presentation [online] 2015. Available at: http://emedicine.medscape.com/ article/1156220-clinical

5. Omuro A, DeAngelis LM. Glioblastoma and other malignant gliomas: a clinical review. JAMA 2013;310:1842-50.

6. Nelson SJ, Cha S. Imaging glioblastoma multiforme. Cancer J 2003;9:134-45.

7. Ohgaki $\mathrm{H}$, Kleihues P. Genetic pathways to primary and secondary glioblastoma. Am J Pathol 2007;170:1445-53.

8. Kanu OO, Hughes B, Di C, Lin N, Fu J, Bigner DD, et al. Glioblastoma Multiforme Oncogenomics and Signaling Pathways. Clin Med Oncol 2009;3:39-52.

9. Garber K. Oncometabolite? IDH1 discoveries raise possibility of new metabolism targets in brain cancers and leukemia. J Natl Cancer Inst 2010;102:926-8.

10. Das PM, Singal R. DNA methylation and cancer. J Clin Oncol 2004;22:4632-42.

11. Krakstad C, Chekenya M. Survival signalling and apoptosis resistance in glioblastomas: opportunities for targeted therapeutics. Mol Cancer 2010;9:135.

12. Ohgaki $\mathrm{H}$, Kleihues $\mathrm{P}$. Genetic alterations and signaling pathways in the evolution of gliomas. Cancer Sci 2009;100:2235-41.

13. Sarkar C, Jain A, Suri V. Current concepts in the pathology and genetics of gliomas. Indian J Cancer 2009;46:108-19.

14. Sathornsumetee S, Rich JN. Designer therapies for glioblastoma multiforme. Ann N Y Acad Sci 2008;1142:108-32. 
15. Esteller M, Garcia-Foncillas J, Andion E, Goodman SN, Hidalgo OF, Vanaclocha V, et al. Inactivation of the DNArepair gene MGMT and the clinical response of gliomas to alkylating agents. N Engl J Med 2000;343:1350-4.

16. Ferroli $P$, Acerbi F, Finocchiaro G. From standard treatment to personalized medicine: role of IDH1 mutations in lowgrade glioma evolution and treatment. World Neurosurg 2010;73:234-6.

17. Bock C, Lengauer T. Computational epigenetics. Bioinformatics 2008;24:1-10.

18. Gopisetty G, Ramachandran K, Singal R. DNA methylation and apoptosis. Mol Immunol 2006;43:1729-40.

19. Robertson KD. DNA methylation and human disease. Nat Rev Genet 2005;6:597-610.

20. Dinçer Y. Tiroid kanserli hastalarda lökosit O6 metil guanin DNA metil transferaz aktivitesinin tayini. [Doktora Tezi], İstanbul: İstanbul Üniversitesi; 2000.

21. Malmer B, Adatto P, Armstrong G, Barnholtz-Sloan J, Bernstein JL, Claus E, et al. GLIOGENE an International Consortium to Understand Familial Glioma. Cancer Epidemiol Biomarkers Prev 2007;16:1730-4.

22. Garber K. Oncometabolite? IDH1 discoveries raise possibility of new metabolism targets in brain cancers and leukemia. J Natl Cancer Inst 2010;102:926-8.

23. Gopisetty G, Ramachandran K, Singal R. DNA methylation and apoptosis. Mol Immunol 2006;43:1729-40.

24. Krakstad C, Chekenya M. Survival signalling and apoptosis resistance in glioblastomas: opportunities for targeted therapeutics. Mol Cancer 2010;9:135.

25. Kloosterhof NK, Bralten LB, Dubbink HJ, French PJ, van den Bent MJ. Isocitrate dehydrogenase-1 mutations: a fundamentally new understanding of diffuse glioma? Lancet Oncol 2011;12:83-91.

26. Yu L, Qi ST, Li ZY. Analysis of isocitrate dehydrogenase-1/2 gene mutations in gliomas. Chin Med J (Engl) 2010;123:3697-705.

27. Sonoda $Y$, Kumabe $T$, Nakamura $T$, Saito $R$, Kanamori $M$, Yamashita Y, et al. Analysis of IDH1 and IDH2 mutations in Japanese glioma patients. Cancer Sci 2009;100:1996-8.

28. von Deimling A, Korshunov A, Hartmann C. The next generation of glioma biomarkers: MGMT methylation, BRAF fusions and IDH1 mutations. Brain Pathol 2011;21:74-87.

29. Koestner A, Swenberg JA, Wechsler W. Transplacental production with ethylnitrosourea of neoplasms of the nervous system in Sprague-Dawley rats. Am J Pathol 1971;63:37-56.
30. Weissenberger J, Steinbach JP, Malin G, Spada S, Rülicke $T$, Aguzzi A. Development and malignant progression of astrocytomas in GFAP-v-src transgenic mice. Oncogene 1997;14:2005-13.

31. Yano H, Hara A, Shinoda J, Takenaka K, Yoshimi N, Mori $\mathrm{H}$, et al. Immunohistochemical analysis of betacatenin in N-ethyl-N-nitrosourea-induced rat gliomas: implications in regulation of angiogenesis. Neurol Res 2000;22:527-32.

32. Liebelt BD, Shingu T, Zhou X, Ren J, Shin SA, Hu J. Glioma Stem Cells: Signaling, Microenvironment, and Therapy. Stem Cells Int 2016;2016:7849890.

33. Plate KH, Breier G, Millauer B, Ullrich A, Risau W. Up-regulation of vascular endothelial growth factor and its cognate receptors in a rat glioma model of tumor angiogenesis. Cancer Res 1993;53:5822-7.

34. Creemers GJ, Lund B, Verweij J. Topoisomerase I inhibitors: topotecan and irenotecan. Cancer Treat Rev 1994;20:73-96.

35. Coggins $C A$, Elion $G B$, Houghton PJ, Hare $C B$, Keir $S$, Colvin OM, et al. Enhancement of irinotecan (CPT-11) activity against central nervous system tumor xenografts by alkylating agents. Cancer Chemother Pharmacol 1998;41:485-90.

36. Vredenburgh JJ, Desjardins A, Herndon JE 2nd, Marcello $J$, Reardon DA, Quinn JA, et al. Bevacizumab plus irinotecan in recurrent glioblastoma multiforme. J Clin Oncol 2007;25:4722-9.

37. Desjardins A, Reardon DA, Herndon JE 2nd, Marcello J, Quinn JA, Rich JN, et al. Bevacizumab plus irinotecan in recurrent WHO grade 3 malignant gliomas. Clin Cancer Res 2008;14:7068-73.

38. Friedman HS, Dolan ME, Pegg AE, Marcelli S, Keir S, Catino $\mathrm{JJ}$, et al. Activity of temozolomide in the treatment of central nervous system tumor xenografts. Cancer Res 1995;55:2853-7.

39. Plowman J, Waud WR, Koutsoukos AD, Rubinstein LV, Moore TD, Grever MR. Preclinical antitumor activity of temozolomide in mice: efficacy against human brain tumor xenografts and synergism with 1,3-bis(2-chloroethyl)-1-nitrosourea. Cancer Res 1994;54:3793-9.

40. O'Reilly SM, Newlands ES, Glaser MG, Brampton M, RiceEdwards JM, Illingworth RD, et al. Temozolomide: a new oral cytotoxic chemotherapeutic agent with promising activity against primary brain tumours. Eur J Cancer 1993;29A:940-2. 